\title{
Optical Techniques for Determination of Normal Shock Position in Supersonic Flows for Aerospace Applications
}

Grigory Adamovsky

Lewis Research Center

Cleveland, Ohio

and

John G. Eustace

John Carroll University

Cleveland, Ohio

Prepared for the

Conference on Optical Testing and Metrology III

sponsored by the Society of Photo-Optical Instrumentation Engineers

San Diego, California, July 8-13, 1990 
Optical techniques for determination of normal shock position

in supersonic flows for aerospace applications

Grigory Adamovsky

National Aeronautics and Space Administration

Lewis Research Center

Cleveland, Ohio 44135

and

John G. Eustace

John Carroll University

Cleveland, Ohio 44118

\section{ABSTRACT}

This paper presents techniques for the quantitative determination of shock position in supersonic flows using direct and indirect methods. It also presents a description of an experimental setup, explains different configurations of shock position sensing systems, and gives some experimental results. All of the methods discussed are analyzed to determine the ease of technology transfer from the laboratory to in-flight operation.

\section{INTRODUCTION}

Shock waves are boundaries created when transitioning from a supersonic flow regime to a subsonic flow regime. Various methods are used to generate shocks, however all shocks can be classified in terms of their mobility as either dynamic or static shocks. Dynamic shocks are found in ballistics, detonation measurements, and other transient applications. ${ }^{1}$ Stationary shocks are found in the study of gas and fluid dynamics, combustion processes, and propulsion.2

Study of shock front generation and control has been carried on for many years in various areas. A special interest pertains to the determination of the position of a normal shock which is generated in a supersonic nozzle. ${ }^{3,4}$ Knowledge of the shock position can be used for real-time flow analyses, ground based and in-flight evaluation of engine performance and control. 'Engine operation is critically dependent upon the position of the normal shock within the inlet to prevent a catastrophic phenomenon known as inlet unstart from occurring.

This paper addresses various optical techniques to determine the normal shock position in a supersonic nozzle, with an emphasis on airborne applications.

\section{REVIEW OF SENSING TECHNIQUES}

Optical. sensing techniques developed to determine the shock position can be grouped into two classes, direct and indirect methods. 


\subsection{Direct Methods}

Direct methods are based on a visualization of the flow using either schlieren, shadowgraph, or interferometric techniques. In the past these methods were used primarily for qualitative analyses. However, utilization of these techniques for quantitative measurements has also been reported. All three direct methods employ the optical properties of density gradients that occur across the shock. However, they are based on different physical phenomena requiring different equations to describe them as well as different optical configurations. Information on this subject can be easily found in textbooks on gas and fluid dynamics. ${ }^{5-7}$

When analyzing the applicability of direct methods to airborne systems, the interferometric and schlieren techniques do not fare well. The interferometric technique suffers from complexity, required stability, and an involved signal processing scheme. The schlieren technique is found unacceptable because of alignment requirements for the knife edge employed in this method. The only technique that may find application in an airborne system is the shadowgraph. In addition to its simplicity, relatively high stability, and low cost, the shadowgraph is better suited for shock visualization because it measures the second derivative of the density. That is, it measures the shock itself where the density changes the most.

Development of a flight prototype normal shock sensor using the shadowgraph technique has recently been reported. 8

\subsection{Indirect Methods}

Indirect methods employ the optical properties of a shock, specifically the variations in the refractive index across its boundary. The variations take place due to the presence of density gradients across the shock boundary and can be described by the Gradstone-Dale equation. ${ }^{9}$ The resultant gradients in the refractive index cause the shock boundary to deflect an oblique laser beam.

Some configurations of sensing systems based on indirect methods are shown in Fig. 1. In systems with a single receiver (See Fig. 1(a)) a light beam from the transmitter illuminates the entrance aperture of the receiver in such a manner that any deflection of the beam by the shock produces displacement at the aperture and results in changes in the signal obtained. In other words a change in the shock position causes misalignment and leads to additional losses.

To improve the system sensitivity two semitransparent mirrors could be positioned in front of the transmitter and receiver, as is shown in Fig. 1(b), forming a Fabry-Perot interferometer. The intensity modulated light beam enters the interferometer which generates an interference pattern at the modulating frequency. Variations in the shock position produce additional losses in the interferometric cavity between two mirrors due to the deflecting effect of the shock. This will result in variations in intensities of the interfering fields and produce a contrast modulation of the interference pattern. 10

In a system which employs a multi-point receiver system, for instance a linear array, (See.Fig. 1(c)), the beam, which has been deflected by the shock, illuminates a sector of the receiver corresponding to the linear displacement of the beam due to the deflection. The receivers can be photodiode or CCD arrays or position sensitive detectors. When an array of elements is used, the position of the deflected 
beam is retrieved from the intensity profile of the illuminated elements. When a position sensitive detector is used, the current from the detector is a function of the relative position of the light beam on the sensitive surface of the detector.

Each of the configurations described above can employ fiber optics to monitor the shock position remotely.

\section{SYSTEM DESCRIPTION}

A block diagram of the experimental setup is shown in Fig. 2. In order to generate a shock, 125 psi air is sent into a specially designed supersonic nozzle $\mathrm{N}$. The nozzle has a 1 in. diameter transparent window centered in the minimum throat region. A drawing of the nozzle is presented in Fig. 3. After passing through the nozzle and an expansion tank $T$ the air escapes into the atmosphere. Two pressure gages P1 and P2 are installed to measure pressures upstream and downstream of the nozzle. For certain values of P1 and P2 the system generates a normal shock downstream of the minimum throat region. P2, the back pressure, can be changed using the pressure regulator $\mathrm{R}$. A change in pressure P2 leads to change in the position of the shock.

In the experimental setup the shock is visualized by a shadowgraph and the pattern is observed at a screen $S$ and also by a CCD array A through a beamsplitter BS1. The shadowgraph used consists of a laser L1, a beam expander BE, mirrors M1 and M2, and two additional components, a focusing lens FL and a knife K. These additional components convert a conventional shadowgraph into a dark field shadowgraph. The dark field shadowgraph forms a bright image of the shock on a dark background contrary to a conventional one which gives a dark image on a bright background.

The principle of operation of the dark field shadowgraph is based on the deflection of a portion of a light beam by the shock. The knife blocks the unperturbed portion of the light beam and permits the deflected portion to pass.

Patterns generated by a conventional and a dark field shadowgraph under the same conditions and detected by a CCD array are shown in Fig. 4(a) and (b) respectively. It is easy to see that the modification eliminates background noise associated with the CCD array. Conversion from a conventional shadowgraph to a dark field one can be easily achieved by appropriate positioning of the knife edge.

The configuration of the system for indirect measurements is based on the principle shown in Fig. 1(a). The system consists (See Fig. 2) of a laser L2, a beamsplitter BS2, a photodetector P, and two mirrors, M3 and M4. A beam from the laser L2 after reflection from the mirrors is sent to the photodetector by the beamsplitter BS2. The test section is located between the mirrors and the laser beam passes through the window in the nozzle at an oblique angle. A shock generated in the nozzle passes through the laser beam and causes the latter to deflect. This deflection results in a linear displacement of the laser beam at the aperture of the photodetector. Since the change in refractive index due to the shock is finite, but small, the angular deflection caused by this change is also small over the range of interest. Thus, the deflection can be detected over a very small region, such as the active area of a single pin photodiode, or the aperture of a single fiber. 


\section{EXPERIMENT}

During the experiment direct and indirect systems were run simultaneously and the data from the direct system were used to calibrate the indirect one. The calibration was done by measuring the position of the shock visualized on the screen of the oscilloscope at selected pressures P1 and P2, correlating the position to the physical dimensions of the transparent window, and relating it to a readout from the photodetector $P$. This procedure was repeated several times and the resulting average values were later used.

In the shadowgraph system the visualized shock was displayed on the screen of an oscilloscope via a CCD array. Data from the system were obtained in terms of the position of the shock from the center of the oscilloscope screen. This was done using the valid assumption that with proper alignment the center of the screen corresponded to the minimum throat region, which was located at the center of the transparent nozzle window. Using the geometrical parameters of the window it was than possible to calculate the actual distance between the shock and the minimum throat region. Data obtained from the shadowgraph are plotted in Fig. 5. This figure shows the shock position as a function of the pressure ratio P2/P1.

In the indirect sensing system a change in the position of the beam resulted in a change in the photodetector output signal. This signal was then recorded as a function of the pressure ratio. The results are plotted in Fig. 6. The graph shows that the shock starts interacting with the beam only after the pressure ratio reaches approximately 0.775 . The system desuribed in this report has a limited range of usefulness for shock position sensing because at pressure ratios less than 0.775 the shock front is not intersected by the laser beam. However this limitation can be overcome by designing the transparent window to an appropriate configuration to cover a given range of pressure ratios.

Figure 7 combines the data from Figs. 5 and 6 by displaying the position of the shock as function of the signal from the photodetector.

The conclusion that can be drawn from the results presented in Figs. 6 and 7 is that two types of sensing systems, a shock proximity sensor and a shock position sensor, could be developed based on the indirect method used in the experiment. A shock proximity sensor would employ the rapid change in the signal level observed when the pressure ratio reaches the critical value (See Fig. 6). A shock position sensor would employ that portion of the curve in Fig. 7 where the displacement is dependent on the signal output.

The data suggest that the response of the shock front to the change in the pressure ratio is a linear one. It can be seen from Fig. 5 that the CCD array data corresponding to a pressure ratio of between 0.65 and 0.85 produces a straight line. The pressure can be read to within 0.25 psi on the pressure gauges. The position of the shock image on the CCD array can be read to the smallest division on the oscilloscope screen (See Fig. 4). This value corresponds to a linear displacement of $0.27 \mathrm{~mm}$. With linear interpolation this might be resolved to a greater degree.

\section{ACKNOWLEDGMENTS}

J.G. Eustace acknowledges the support of the NASA Lewis Research Center (NASA Grant NAG-3-984). 


\section{REFERENCES}

1. D.S. Dosanjh, Modern Optical Methods in Gas Dynamic Research, Plenum Press, New York, 1971.

2. R.J. Goldstein, ed., Fluid Mechanics Measurements, Hemisphere Publishing Corp., 1983

3. M. Sajben and R.C. Crites, "Real-Time Optical Measurement of Time-Dependent Shock Position," AIAA J. 17, (8), 910-912, (1979).

4. M. Sajben, J.F. Donovan, and M.J. Morris, "Flight Prototype Shock Sensor,"

(McDonnell Douglas Missile Systems Co., NASA Contract NAS3-25446) NASA CR-185205, 1990 .

5. A.H. Shapiro, The Dynamics and Thermodynamics of Compressible Fluid Flow, Vols. I and II, The Ronald Press Co., New York, 1954.

6. A.B. Cambel and B.H. Jennings, Gas Dynamics, Dover Publications, New York, 1958.

7. W. Merzkirch, Flow Visualization, Academic Press, 0rlando, 1987.

8. D.G. Iverson, Jr. and T.D. Daiber, "Flight Prototype Normal Shock Sensor,"

(Boeing Military Airplane Division, NASA Contract NAS3-25447) NASA CR-185204, 1989.

9. W.L. Howes, "Optical Elements Formed by Compressed Gases," NASA Technical Paper TP-2555, 1986 .

10. G. Adamovsky, T.N. Sherer, and D.T. Maitland, "Intensity-Based Fibre-0ptic Sensing System Using Contrast Modulation of Subcarrier Interference Pattern," Electron Lett. 25, (3), 325-325 (1989). 


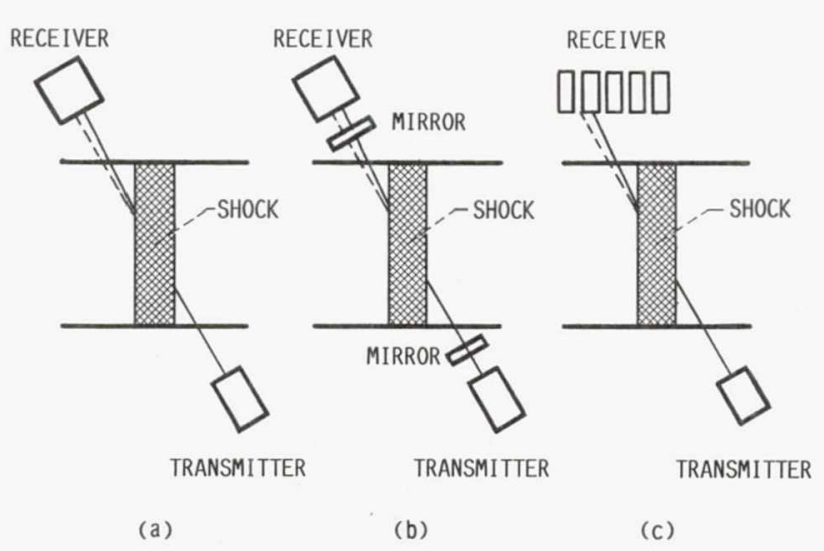

FIGURE 1. - INDIRECT SHOCK POSITION SENSING METHODS INVOLVING LIGHT BEAM DEFLECTION AND

(a) SINGLE RECEIVER,

(b) SINGLE RECEIVER AND SEMITRANSPARENT MIRRORS,

(c) MULTI-POINT RECEIVER.

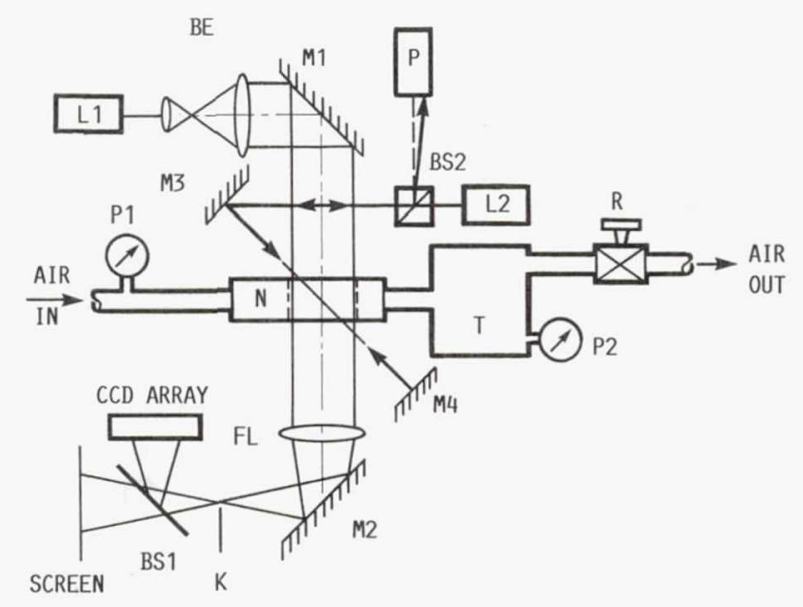

FIGURE 2. - BLOCK DIAGRAM OF THE EXPERIMENTAL SETUP.

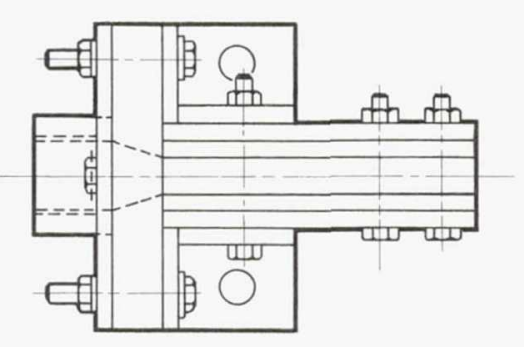

PLAN VIEW

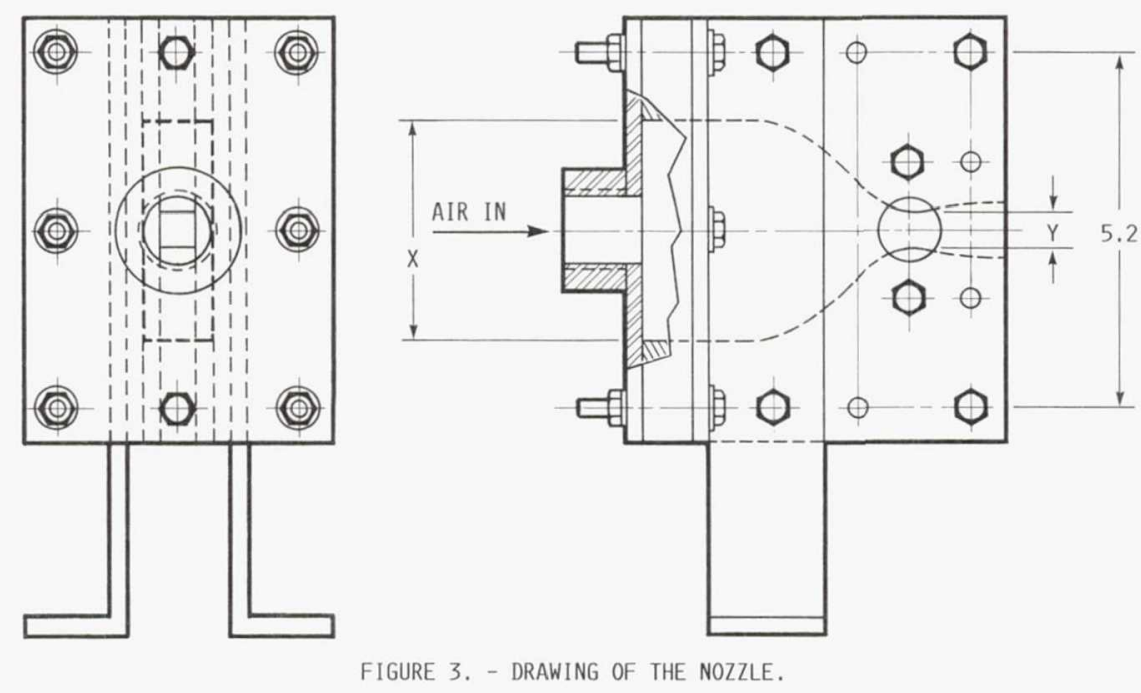



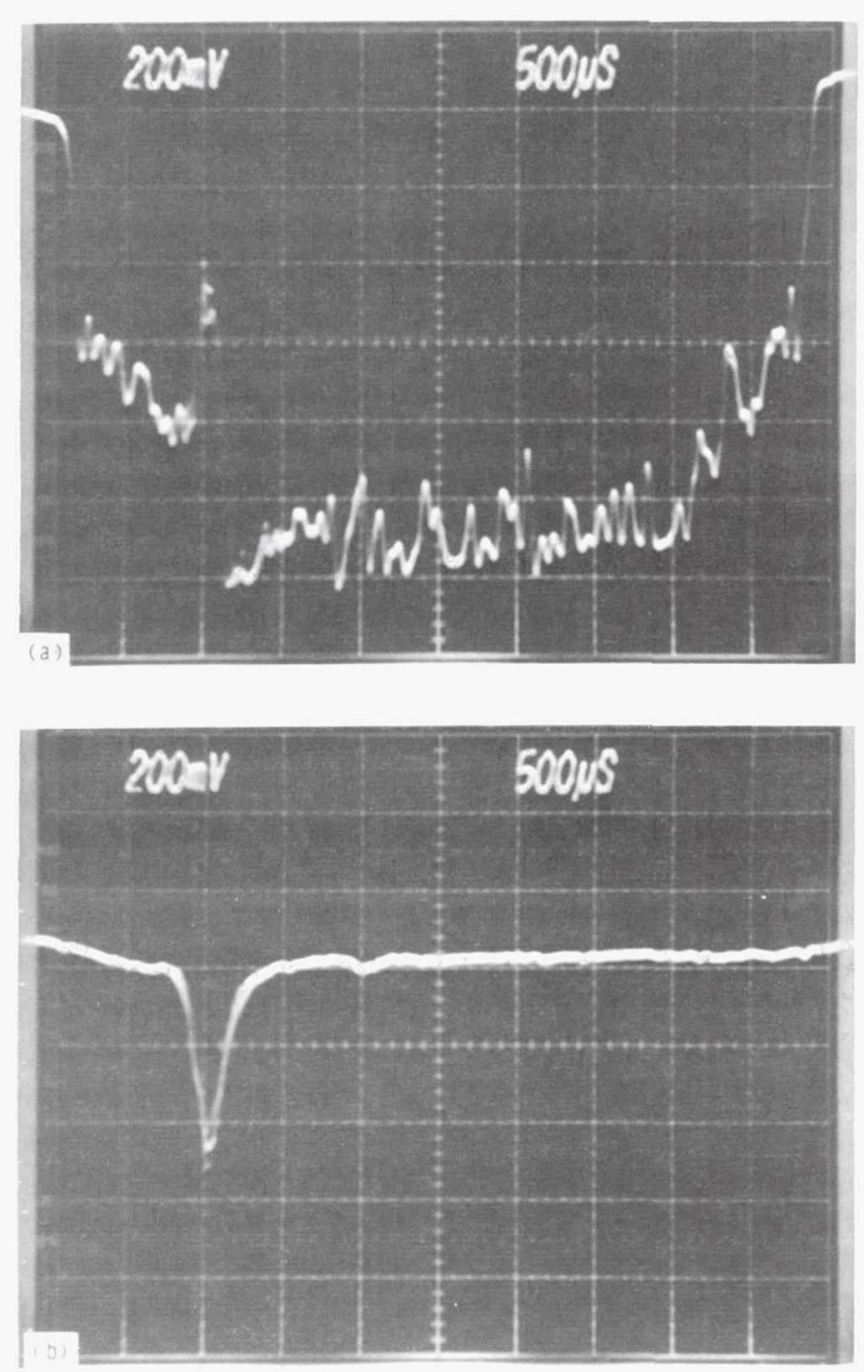

FIGURE 4. - FIELD SEEN BY A CCD ARRAY AND GENERATED BY (a) CONVENTIONAL SHADOWGRAPH.

(b) DARK FIELD SHADOWGRAPH.

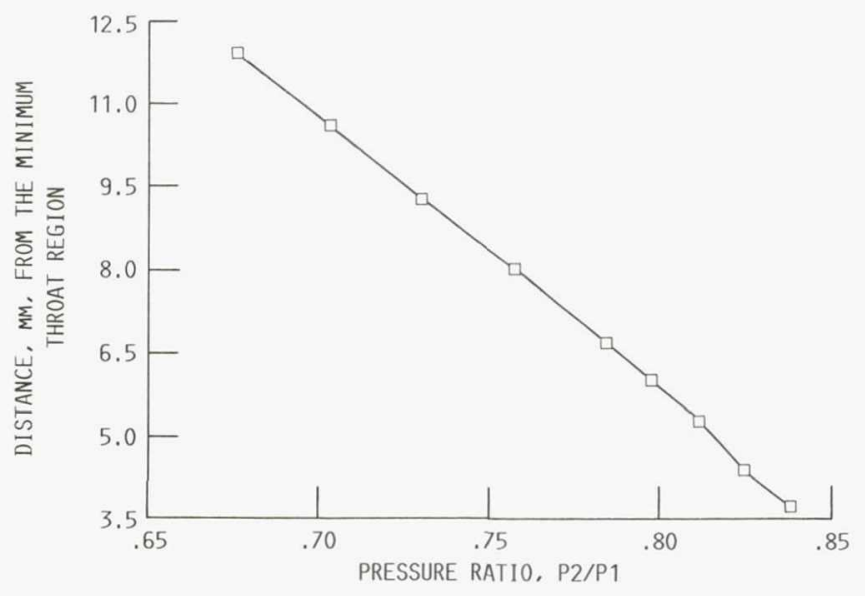

FIGURE 5. - POSITION OF THE SHOCK AS A FUNCTION OF THE PRESSURE RATIO P2/P1 OBTAINED FROM THE SHADOWGRAPH.

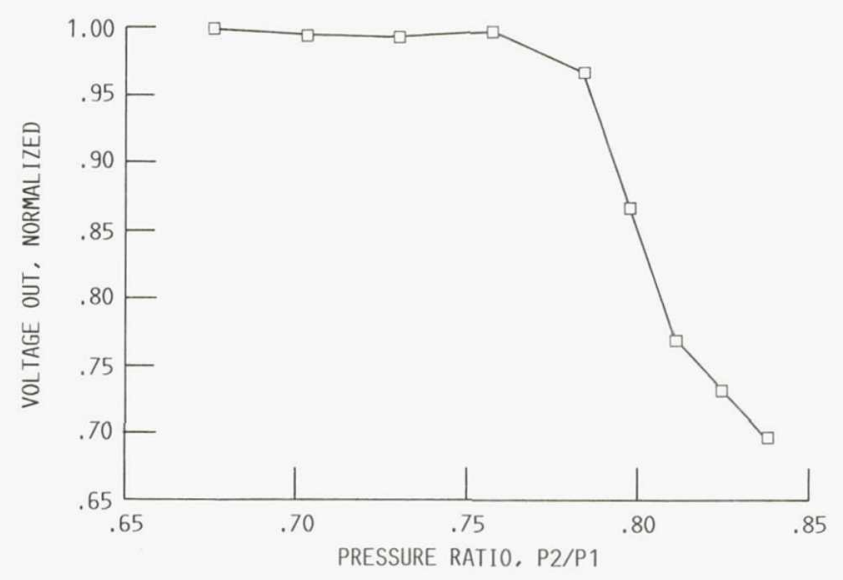

FIGURE 6. - SIGNAL OUT AS A FUNCTION OF THE PRESSURE RATIO P2/P1 OBTAINED USING THE INDIRECT METHOD.

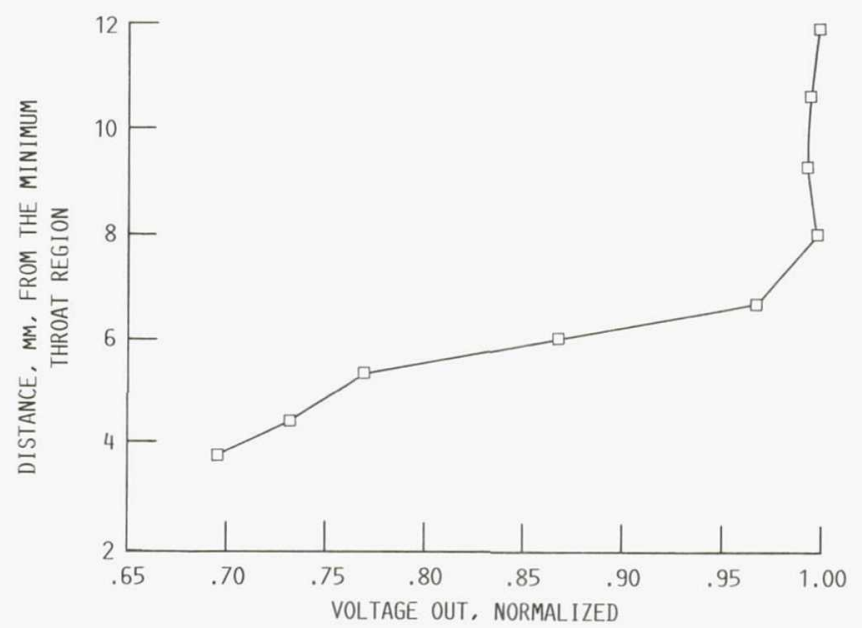

FIGURE 7. - POSITION OF THE SHOCK AS A FUNCTION OF A SIGNAL OUT USING THE INDIRECT METHOD. 


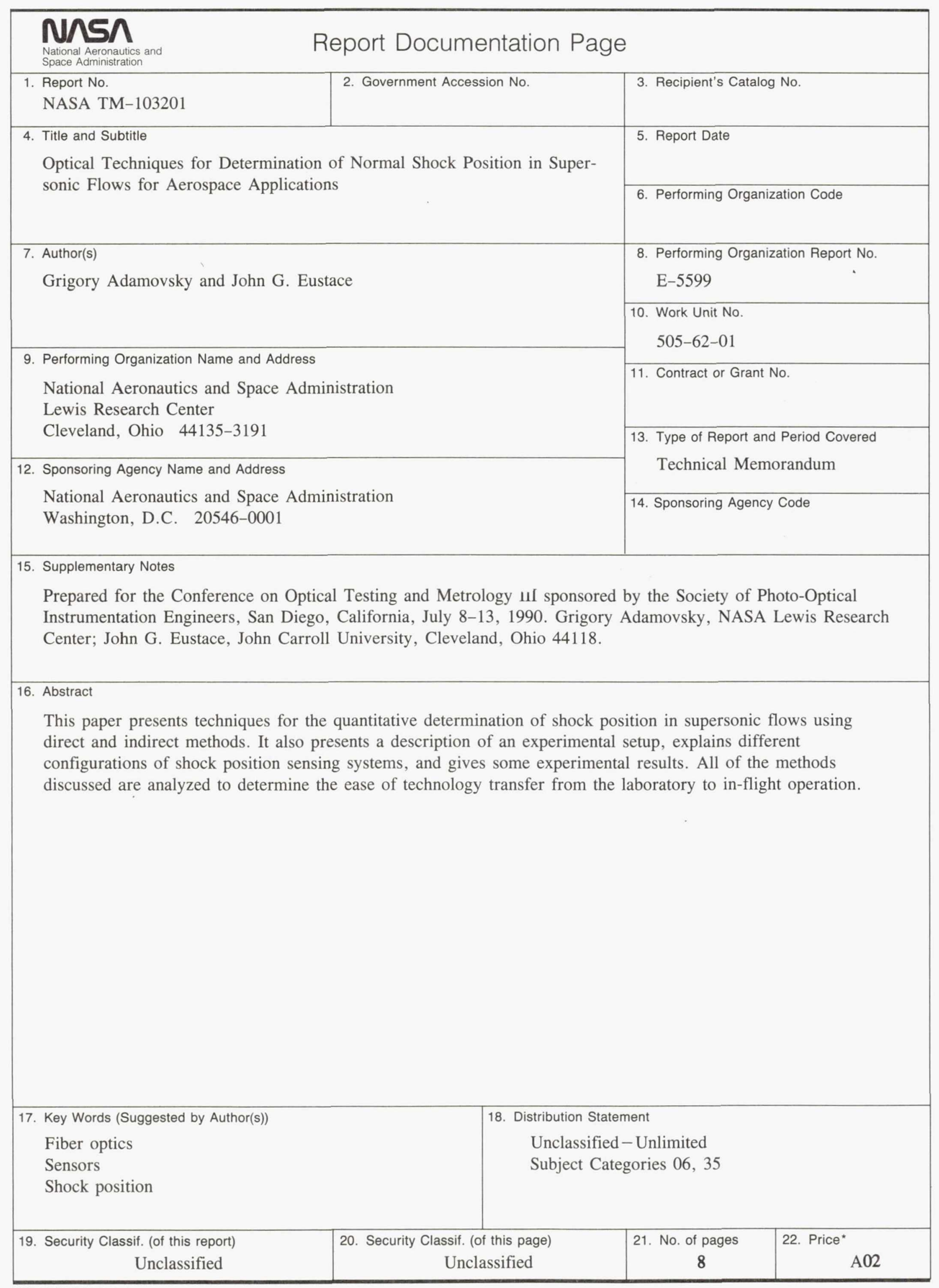

